\title{
Rotationally resolved ultraviolet spectra of benzene-noble gas van der Waals clusters
}

\author{
Th. Weber, E. Riedle, and H.J. Neusser \\ Institut für Physikalische und Theoretische Chemie, Technische Universität München, Lichtenbergstrasse 4, W-8046 Garching, \\ Federal Republic of Germany
}

Received 10 September 1990

\begin{abstract}
Sub-Doppler electronic spectra with hundreds of resolved rotational lines are now available for benzeneAr dimers and trimers. From their analysis the structure of these clusters is precisely determined. The analysis of two bands, $6_{0}^{1}$ and $16_{0}^{2}$, of $\mathrm{C}_{6} \mathrm{H}_{6} \cdot \mathrm{Ar}$ is presented in detail. It leads to accurate values of the van der Waals bond length in the electronic ground and excited state. The change in frequency upon clustering is found to be a factor of 17 larger for the overtone of the out-of-plane mode $v_{16}$ than for the in-plane vibration $v_{1}$. This can be tentatively explained by an interaction of the low frequency out-ofplane motion of the ring with the van-der-Waals motion of the Ar atom.
\end{abstract}

PACS: $33.20 . \mathrm{Lg} ; 35.20 . \mathrm{Pa} ; 36.40 .+\mathrm{d}$

\section{Introduction}

In the past, rotationally resolved spectroscopy has been proven to be an important method for the structural analysis of molecules. During the last decade microwave spectroscopy and rotationally resolved IR spectroscopy have been successfully extended to the investigation of small clusters. There is, however, an apparent lack of rotationally resolved spectroscopic information on clusters of polyatomic, particularly organic molecules. This is mainly due to the often small dipole moments of these clusters and the inherent complex and dense structure of the spectra. Particularly for large clusters the fundamental question arises whether these clusters display a stable structure on the time scale of the experiment or whether a rapid isomerization or tunneling does not allow to determine a single defined geometry.

In this paper we focus attention on benzene-noble gas clusters. Microwave and IR spectra of benzene-noble gas clusters are not yet available and UV spectroscopy appears to be a feasible method if the dense rotational structure of the vibronic bands can be resolved. The necessary spectral resolution can now be obtained, as shown in our recent work [1].

\section{Experimental}

In a supersonic cooled beam of large molecules a variety of clusters is produced, whose UV spectra generally overlap. In this work the technique of resonance-enhanced two-photon ionization [2] is used for mass and species selection in combination with a frequency-doubled pulsed amplified $\mathrm{cw}$ laser source. The benzene-Ar clusters are produced in a skimmed supersonic jet expansion of a mixture of $2-5$ bar Ar and $40 \mathrm{mbar}^{\mathrm{C}} \mathrm{H}_{6}$. Due to the skimmer the Doppler width is reduced to about $50 \mathrm{MHz}$ at the transition frequency of $38585 \mathrm{~cm}^{-1}$. The clusters are excited to a particular vibration in the $S_{1}\left({ }^{1} B_{2 u}\right)$ state by absorption of a photon from the narrow bandwidth $(\Delta v \approx 100 \mathrm{MHz}$ ) light beam. Ionization occurs after the absorption of a second photon from a frequency-doubled dye laser (FL 2002, Lambda Physik) with a relatively broad frequency-width of $0.5 \mathrm{~cm}^{-1}$. The wavelength of the second laser is chosen so that the excess energy above the ionization potential of the benzene-Ar cluster is some $700 \mathrm{~cm}^{-1}$. This value is a compromise in order to avoid dissociation of the cluster on the one hand and to obtain a sufficiently strong ion-signal on the other hand. The ions are mass separated in a home built Wiley-McLaren type time-of-flight mass spectrometer. Overview spectra at a low resolution of $0.5 \mathrm{~cm}^{-1}$ are measured in a one-colour experiment, with the FL 2002 dye laser.

\section{Results}

In Fig. 1 a $140 \mathrm{~cm}^{-1}$ overview section of the intermediate state spectrum around the $60_{0}^{1}$ band of the benzene-Ar cluster is shown. Here the ion current at 118 [a.u.], i.e. the mass of $\mathrm{C}_{6} \mathrm{H}_{6} \cdot \mathrm{Ar}$, was monitored. The upper trace represents the same spectrum measured with higher laser intensity. The blue part of the spectrum was already 


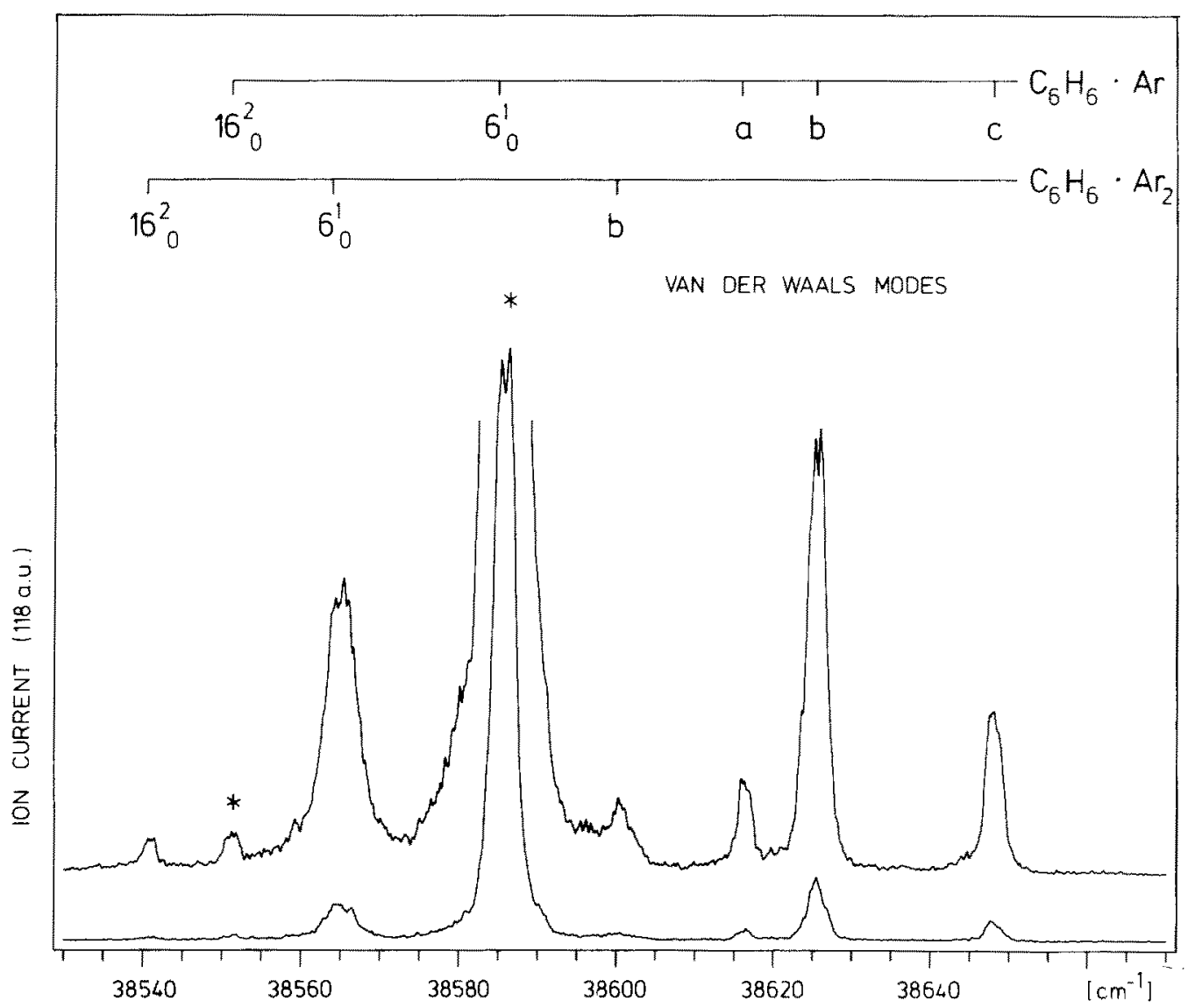

Fig. 1. Low resolution intermediate state spectrum in the region of the $6{ }_{0}^{1}$ band of the $\mathrm{C}_{6} \mathrm{H}_{6} \cdot \mathrm{Ar}$ cluster for two laser intensities, monitored by the ion-current at the $\mathrm{C}_{6} \mathrm{H}_{6} \cdot \operatorname{Ar}$ mass (118 [a.u.]). The assignment of the transitions is given at the top; $a, b, c$ designate van der Waals modes. Peaks that are rotationally analyzed in this work are marked by asterisks

presented by Menapace et al. [3], however, their spectrum contained a dip at the position of the monomer $6_{0}^{1}$ band due to saturation of the detector. This is avoided in our setup by repulsion of the $\mathrm{C}_{6} \mathrm{H}_{6}^{+}$ions [1]. The strong peak at $38585 \mathrm{~cm}^{-1}$ is easily identified as the $6_{0}^{1}$ band of $\mathrm{C}_{6} \mathrm{H}_{6} \cdot \operatorname{Ar}[4]$ but within the observed spectral range 7 additional weaker peaks appear whose origin is not a pirori clear. On top of Fig. 1 the assignment of all peaks is given. This assignment was obtained from the structure of rotationally resolved spectra of the corresponding bands and from a comparison of the rotationally resolved spectra measured simultaneously at the mass of $\mathrm{C}_{6} \mathrm{H}_{6} \cdot \operatorname{Ar}(118$ [a.u.]) and the mass of $\mathrm{C}_{6} \mathrm{H}_{6} \cdot \mathrm{Ar}_{2}$ (158 [a.u.]). Even though only ions with 118 [a.u.] are monitored in the spectrum several bands of $\mathrm{C}_{6} \mathrm{H}_{6} \cdot \mathrm{Ar}_{2}(158$ [a.u.]) appear. From this it is concluded that a dissociation of the $\left(\mathrm{C}_{6} \mathrm{H}_{6} \cdot \mathrm{Ar}_{2}\right)^{+}$ions occurs in the laser focus (ion source) of the time-of-flight mass spectrometer and ions originally produced as $\left(\mathrm{C}_{6} \mathrm{H}_{6} \cdot \mathrm{Ar}_{2}\right)^{+}$are monitored as a daughter ion of the following dissociation process:

$$
\begin{array}{ll}
\left(\mathrm{C}_{6} \mathrm{H}_{6} \cdot \mathrm{Ar}_{2}\right)^{+} \rightarrow & \left(\mathrm{C}_{6} \mathrm{H}_{6} \cdot \mathrm{Ar}\right)^{+}+\mathrm{Ar} \\
158 \text { [a.u.] } & 118 \text { [a.u.] }
\end{array}
$$

A dissociation of neutral $\mathrm{C}_{6} \mathrm{H}_{6} \cdot \mathrm{Ar}_{2}$ in $\mathrm{C}_{6} \mathrm{H}_{6} \cdot \mathrm{Ar}+\mathrm{Ar}$ and a subsequent ionization of $\mathrm{C}_{6} \mathrm{H}_{6} \cdot \mathrm{Ar}$ is another possibil- ity. It must have occurred during the laser pulse and thus would lead to a broadening of the lines. This dissociation process complicates the analysis and assignment of the low resolution mass spectrum and requires some additional arguments for analysis.

A clear distinction of the different contributions is possible on the basis of the resolved rotational structure of the bands. The analysis of the $\mathrm{C}_{6} \mathrm{H}_{6} \cdot \mathrm{Ar}_{2}$ bands will be given elsewhere [5]. Briefly, it allows to determine a definite structure of the Benzene- $\mathrm{Ar}_{2}$ cluster and to find an accurate value for the van der Waals bond lengths in the trimer. Here we would like to illustrate the rotational analysis for two bands of $\mathrm{C}_{6} \mathrm{H}_{6} \cdot \mathrm{Ar}$, i.e. the $6_{0}^{1}$ and $16_{0}^{2}$ band marked by asterisks in Fig. 1. They are examples for in-plane and out-of-plane vibrations of the benzene ring, respectively. Thus the interesting question can be addressed, whether the out-of-plane character of a vibration influences the van der Waals bonding.

Although the intensity of the $16_{0}^{2}$ band is only $1 \%$ of the $6_{0}^{1}$ band (see Fig. 1) we were able to measure the rotationally resolved spectrum of this weak transition with good signal-to-noise ratio. As shown in Fig. 2, the general appearance of the rotational structure of the two bands is quite different. Both spectra display a $P_{-}$and $R$ branch with widely spaced lines at the red and the blue side, respectively. The $Q$-branch is located in the center 


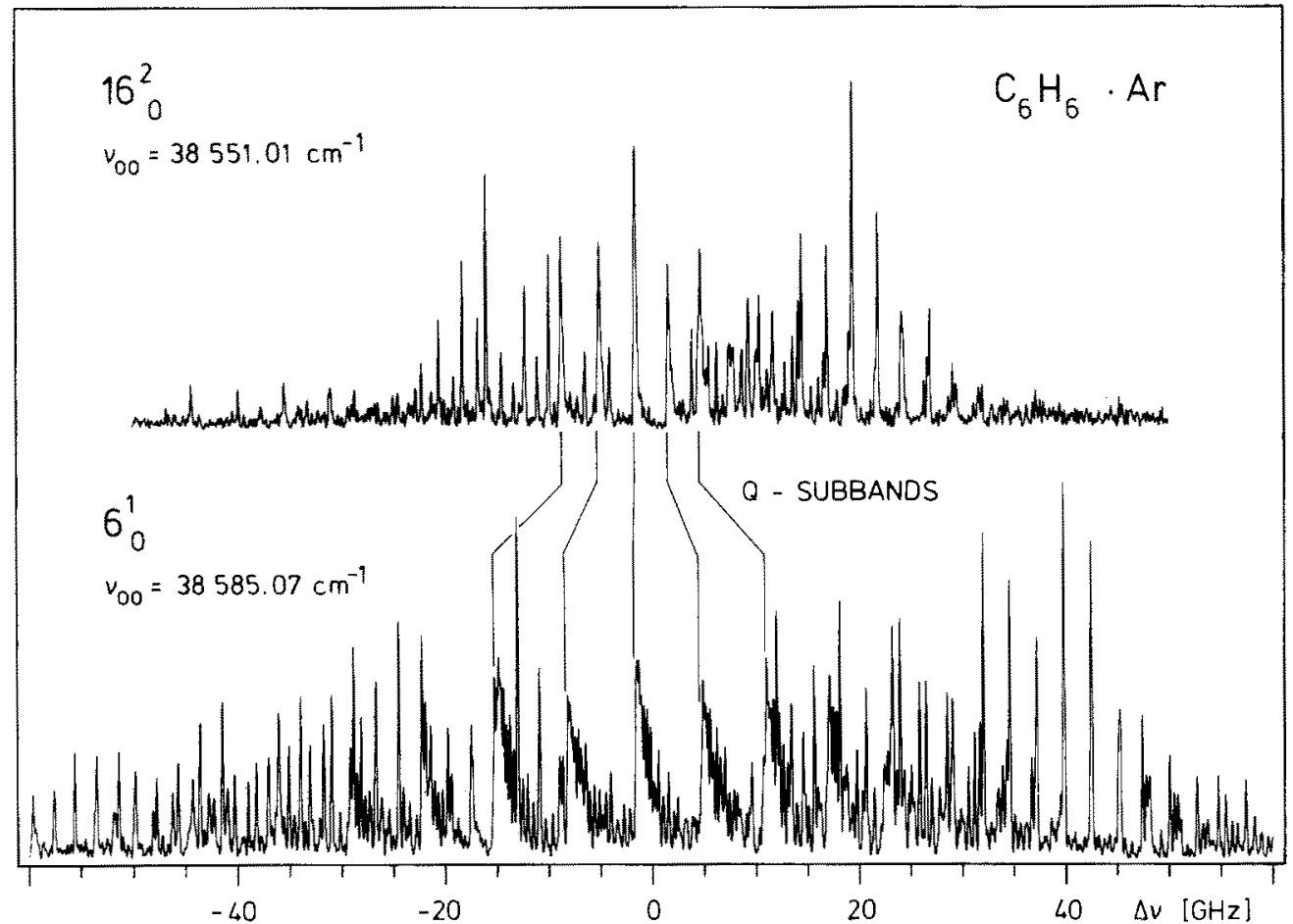

Fig. 2. Rotationally resolved mass-selected resonance-enhanced two-photon ionization spectra of the $6_{0}^{1}$ and the $16_{0}^{2}$ bands of benzene-Ar. For illustration of the differing structure, the $Q$-subbands with equal values of $K$ and $\Delta K$ are connected by lines

Table 1. Spectroscopic constants of the $6_{0}^{1}$ and $16_{0}^{2}$ bands of the $\mathrm{C}_{6} \mathrm{H}_{6}$ monomer and the $\mathrm{C}_{6} \mathrm{H}_{6} \cdot \mathrm{Ar}$ complex leading to the averaged distance $\left\langle r_{\mathrm{Ar}}\right\rangle$ between the Ar atom and the benzene ring. $v_{00}$ is the frequency of the rotationless origin of each band. $A, B$ and $C$ are the rotational constants and $\xi_{\text {eff }}$ is the Coriolis coupling constant. $N$ is the number of assigned unblended rovibronic lines and $\sigma$ the standard deviation of the fit

\begin{tabular}{|c|c|c|c|c|}
\hline & \multicolumn{2}{|l|}{$6_{0}^{1 a}$} & \multicolumn{2}{|l|}{$16_{0}^{2}$} \\
\hline & $\mathrm{C}_{6} \mathrm{H}_{6}$ & $\mathrm{C}_{6} \mathrm{H}_{6} \cdot \mathrm{Ar}$ & $\mathrm{C}_{6} \mathrm{H}_{6}$ & $\mathrm{C}_{6} \mathrm{H}_{6} \cdot \mathrm{Ar}$ \\
\hline$v_{00}\left[\mathrm{~cm}^{-1}\right]$ & $38606.089(8)$ & $38585.071(8)$ & $38560.794(9)$ & $38551.005(4)$ \\
\hline$C_{0}^{\prime \prime} / A_{0}^{\prime \prime}\left[\mathrm{cm}^{-1}\right]$ & 0.0948809 & 0.0948809 & 0.0948809 & 0.0948809 \\
\hline$B_{0}^{\prime \prime}\left[\mathrm{cm}^{-1}\right]$ & $0.189762(2)$ & $0.039371(8)$ & $0.189762(2)$ & $0.039357(24)$ \\
\hline$C_{v}^{\prime} / A_{v}^{\prime}\left[\mathrm{cm}^{-1}\right]$ & $0.090865(3)$ & $0.090862(5)$ & $0.091212(5)$ & $0.091194(13)$ \\
\hline$B_{v}^{\prime}\left[\mathrm{cm}^{-1}\right]$ & $0.181778(2)$ & $0.040060(8)$ & $0.181660(4)$ & $0.039728(21)$ \\
\hline$\zeta_{\text {eff }}^{\prime}$ & $-0.5785(5)$ & $-0.5853(6)$ & $+0.0008(2)$ & $-0.0055(3)$ \\
\hline$N$ & 353 & 226 & 81 & 53 \\
\hline$\sigma[\mathrm{MHz}]$ & 13.9 & 26.8 & 18.4 & 22.1 \\
\hline$\left\langle r_{\mathrm{Ar}}^{\prime \prime}\right\rangle[\AA]$ & & $3.583(1)$ & & $3.584(2)$ \\
\hline$\left\langle r_{\mathrm{Ar}}^{\prime}\right\rangle[\AA]$ & & $3.523(2)$ & & $3.542(3)$ \\
\hline
\end{tabular}

a Taken from [1]

and is divided into blue-shaded subbranches. The striking difference is, that the $6{ }_{0}^{1}$ band is spread over a much larger frequency range than the $16_{0}^{2}$ band. This compression is caused by a reduction of the distance between the lines (i.e., the $Q$-subbands in Fig. 2) due to the nearly zero Coriolis coupling constant $\zeta$ of the out-of-plane mode $v_{16}$. Additionally, the $Q$-subbands of the $16_{0}^{2}$ band are less blue shaded, which indicates that $\Delta B=\left(B_{v}^{\prime}-B_{0}^{\prime \prime}\right)$ is still positive but smaller than for the $6_{0}^{1}$ band (see below).

The rotational constants can be precisely determined by a detailed assignment of the rotational lines and a computer fit to the observed line positions according to the energy predicted for a rigid prolate symmetric top:

$E_{\text {rol }}=B \cdot J \cdot(J+1)+(A-B) \cdot K^{2} \mp 2 \cdot A \cdot \zeta_{\text {eff }} \cdot K$

The selection rules are $\Delta J=0, \pm 1$ and $\Delta K= \pm 1$ (perpendicular transition). The rotational constants are listed together with the benzene data in Table 1 . For the fit the $A_{0}^{\prime \prime}$ constant of $\mathrm{C}_{6} \mathrm{H}_{6} \cdot \mathrm{Ar}$ was fixed to the $C_{0}^{\prime \prime}$ value of $\mathrm{C}_{6} \mathrm{H}_{6}$ as before [1]. This is reasonable, as the value of $A_{v}^{\prime}$ for $\mathrm{C}_{6} \mathrm{H}_{6} \cdot \mathrm{Ar}$ from the fit is equal to $C_{v}^{\prime}$ of $\mathrm{C}_{6} \mathrm{H}_{6}$ and the ground state constants $B_{0}^{\prime \prime}$ found from the fit of the $16_{0}^{2}$ 
and $6_{0}^{1}$ bands are the same within the experimental uncertainty. Additionally, for both bands a difference of +0.007 for $\zeta_{\text {eff }}^{\prime}$ of the monomer and the dimer is found. These results corroborate the assignment of the $16_{0}^{2}$ band of $\mathrm{C}_{6} \mathrm{H}_{6} \cdot$ Ar.

From the fact, that the spectra can be well fitted by a symmetric top Hamiltonian it is concluded that the average position of the $\mathrm{Ar}$ atom is above the benzene plane on the $\mathrm{C}_{6}$ rotational axis. The averaged distance $\left\langle r_{\mathrm{Ar}}\right\rangle$ between the Ar atom and the benzene plane in the ground as well as in the excited electronic state was calculated from the rotational constants $B_{0}^{\prime \prime}$ and $B_{v}^{\prime}$ and is also listed in Table 1 . The average bond length $\left\langle r_{\mathrm{Ar}}^{\prime}\right\rangle$ in the $S_{1}$ state as determined from the $16_{0}^{2}$ band is $20 \mathrm{~mA}$ larger than the corresponding value from the $6_{0}^{1}$ band. This apparent difference can be qualitatively explained by the out-ofplane character and the low frequency $\left(237 \mathrm{~cm}^{-1}\right)$ of the $v_{16}$. It leads to large amplitude motions of the $\mathrm{C}$ and $\mathrm{H}$ atoms out of the ring plane and to a slightly larger average distance of the Ar atom from the plane.

The rotational analysis yields an accurate value for the rotationless transition frequencies. We find $38585.07 \mathrm{~cm}^{-1}$ for the $6{ }_{0}^{1}$ band and $38551.01 \mathrm{~cm}^{-1}$ for the $16_{0}^{2}$ band. The red shift of the $6_{0}^{1}$ transition in $\mathrm{C}_{6} \mathrm{H}_{6} \cdot \mathrm{Ar}$ with respect to $\mathrm{C}_{6} \mathrm{H}_{6}$ is $21.018 \mathrm{~cm}^{-1}$ whereas the red shift of the $16_{0}^{2}$ transition is only $9.789 \mathrm{~cm}^{-1}$. This result is now compared with the red shift of $20.428 \mathrm{~cm}^{-1}$ of the $6{ }_{0}^{1} 1_{0}^{1}$ band [1]. We find a striking stronger change of the red shift for the $v_{16}$ vibrational mode. Most likely this is caused by the out-of-plane character of the $v_{16}\left(e_{2 u}\right)$ vibration, which might interact with the van der Waals motion of the Ar atom.

\section{Conclusion}

We have shown that the rotational analysis of clusters of large molecules with noble gases is possible by high resolution sub-Doppler UV spectroscopy in a cooled supersonic beam. Even for mass selected resonanceenhanced two-photon ionization the measured spectra are a superposition of spectra from different clusters. We showed for benzene-Ar that the complex structure of the low resolution spectrum can be completely analyzed when the bands are rotationally resolved. The rotational analysis of the $16_{0}^{2}$ and $6_{0}^{1}$ bands leads to precise rotational constants and precise values for the averaged van der Waals bond length. The out-of-plane character of the $v_{16}$ mode causes a surprisingly strong frequency shift of the $16_{0}^{2}$ band in the cluster. This points to an interaction of the out-of-plane motion in the benzene ring and the van der Waals vibrational motion of the Ar atom.

\section{References}

1. Weber, Th., von Bargen, A., Riedle, E., Neusser, H.J.: J. Chem. Phys, 92, 90 (1990)

2. Boesl, U., Neusser, H.J., Schlag, E.W.: J. Am. Chem. Soc. 103, 5058 (1981)

3. Menapace, J.A., Bernstein, E.R.: J. Phys. Chem. 91, 2533 (1987)

4. Fung, K.H., Selzle, H.L., Schlag, E.W.: Z. Naturforsch. 36a, 1338 (1981)

5. Weber, Th., Neusser, H.J.: J. Chem. Phys. (submitted for publication) 